\title{
Reply to Lolli et al
}

\section{Joseph O.C. Coyne, Sophia Nimphius, Robert U. Newton, and G. Gregory Haff}

We thank Lolli et $\mathrm{al}^{1}$ for their comment on our recent article "Does mathematical coupling matter to the acute to chronic workload ratio? A case study from elite sport."2 In their comment, Lolli et $\mathrm{al}^{1}$ highlighted the potential of psuedoreplication ${ }^{3}$ in our paper's statistical analysis. We agree that we had fallen foul of this analytical pitfall and appreciate their identification of this issue. We have since reanalyzed the data using what we consider more appropriate measures for within-subject analysis to address the overall statistical concerns including psuedoreplication. Our analysis now includes examining the repeated-measures correlations ${ }^{4}$ (as suggested by Lolli et $\mathrm{al}^{1}$ ) between (1) acute training load (ATL) and coupled and uncoupled chronic training load (CTL) and (2) coupled and uncoupled acute-to-chronic workload ratios (ACWR). These repeated-measures correlations between coupled ATL-CTL and uncoupled ATL-CTL have also been compared against one another with $r-z$ transformations. The results are presented in Table 1. In addition, we extended the statistical revision to evaluate the outcome using linear mixed models (with the athlete and the time point [day] as random intercept) to determine if there are significant differences between coupled and uncoupled CTL and coupled and uncoupled ACWR. Effect sizes of these differences (marginal $\left.f^{2}\right)^{5}$ were then calculated and interpreted as trivial $(<0.02)$, small $(0.02-0.14)$, medium $(0.15-$ $0.34)$, and large $(>0.35)^{6}$ as presented in Table 2. All statistical analyses were performed using the $\mathrm{R}$ statistics package (https:// www.r-project.org; R Foundation for Statistical Computing, Vienna, Austria), and our $\mathrm{R}$ script has been provided as Supplementary Material (available online).

The key findings of this reanalysis suggest that there may not be a natural correlation between ATL and CTL irrespective of any coupling in the calculation of the CTL with the current real data. This is contrary to what our original article suggested. However, despite significant differences between coupled and uncoupled CTL (in all but open-skill internal and external training load [TL] as an exponentially weighted moving average), the effect sizes of these differences appear trivial $\left(f^{2}<\right.$ 0.02). There also appear to be very large to nearly perfect correlations between coupled and uncoupled ACWR. Similar to CTL, the differences between coupled and uncoupled ACWR, where significant, appear trivial. This is similar to what we reported in our original article. It is also consistent with our original interpretation that mathematical coupling had little effect on practical measures of the ACWR in this case study despite the nature of the sport, training-load calculation method, or whether internal or external load was used.

The authors are with the Centre for Exercise and Sports Science Research, School of Medical and Health Sciences, Edith Cowan University, Joondalup, WA, Australia. Nimphius is also with the Sports Performance Research Inst New Zealand (SPRINZ), University of Technology, Auckland, New Zealand. Haff is also with Sport, Exercise and Physiotherapy, University of Salford, Salford, United Kingdom. Coyne (coach@josephcoyne.com) is corresponding author.
However, we also acknowledge there may be other critical conceptual and statistical issues with the ACWR and the previous literature using the ratio as a measure. As such, we continue to recommend that practitioners be wary of applying the ACWR, and it should not be used in isolation for athlete monitoring. ${ }^{7}$ Practitioners wanting to apply a measure of change in training load are encouraged to investigate other potential options (eg, differential $\operatorname{load}^{8}$ or training stress balance $\left.{ }^{9}\right)$ that may not have the issues associated with using a ratio when trying to determine whether relationships exist with performance or injury. Finally, we wish to thank Lolli et $\mathrm{al}^{1}$ for their diligence in identifying our statistical oversight in the original article, and we look forward to future discussion on this topic.

\section{Acknowledgments}

The Australian Centre for Research into Injury in Sport and its Prevention (ACRISP) at Edith Cowan University is one of the international research centers for the prevention of injury and protection of athlete health supported by the International Olympic Committee. We thank Shih Ching $\mathrm{Fu}$ for statistical assistance with the linear mixed models in R.

\section{References}

1. Lolli L, Batterham AM, MacMillan G, Gregson W, Atkinson G. A comment on "Does mathematical coupling matter to the acute to chronic workload ratio? A case study from elite sport." Int J Sports Physiol Perform. 2020;15(5):600. doi:10.1123/ijspp.2019-0949

2. Coyne JOC, Nimphius S, Newton RU, Haff GG. Does mathematical coupling matter to the acute to chronic workload ratio? A case study from elite sport. Int J Sports Physiol Perform. 2019;14(1):14471454. doi:10.1123/ijspp.2018-0874

3. Lazic SE. The problem of pseudoreplication in neuroscientific studies: is it affecting your analysis? BMC Neurosci. 2010;11:5. PubMed ID: 20074371 doi:10.1186/1471-2202-11-5

4. Bakdash JZ, Marusich LR. Repeated measures correlation. Front Psychol. 2017;8:456. PubMed ID: 28439244 doi:10.3389/fpsyg. 2017.00456

5. Aiken LS, West SG. Multiple Regression: Testing and Interpreting Interactions. Newbury Park, CA: Sage; 1991.

6. Cohen J. A power primer. Psychol Bull. 1992;112(1):155-159. PubMed ID: 19565683 doi:10.1037/0033-2909.112.1.155

7. Weaving D, Jones B, Till K, Abt G, Beggs C. The case for adopting a multivariate approach to optimize training load quantification in team sports. Front Physiol. 2017;8:1024. PubMed ID: 29311959 doi:10. 3389/fphys.2017.01024

8. Lazarus BH, Stewart AM, White KM, et al. Proposal of a global training load measure predicting match performance in an elite team sport. Front Physiol. 2017;8:930. PubMed ID: 29209229 doi:10. 3389/fphys.2017.00930

9. Allen H, Coggan A. Training and Racing With a Powermeter. 2nd ed. Boulder, CO: Velopress; 2010. 


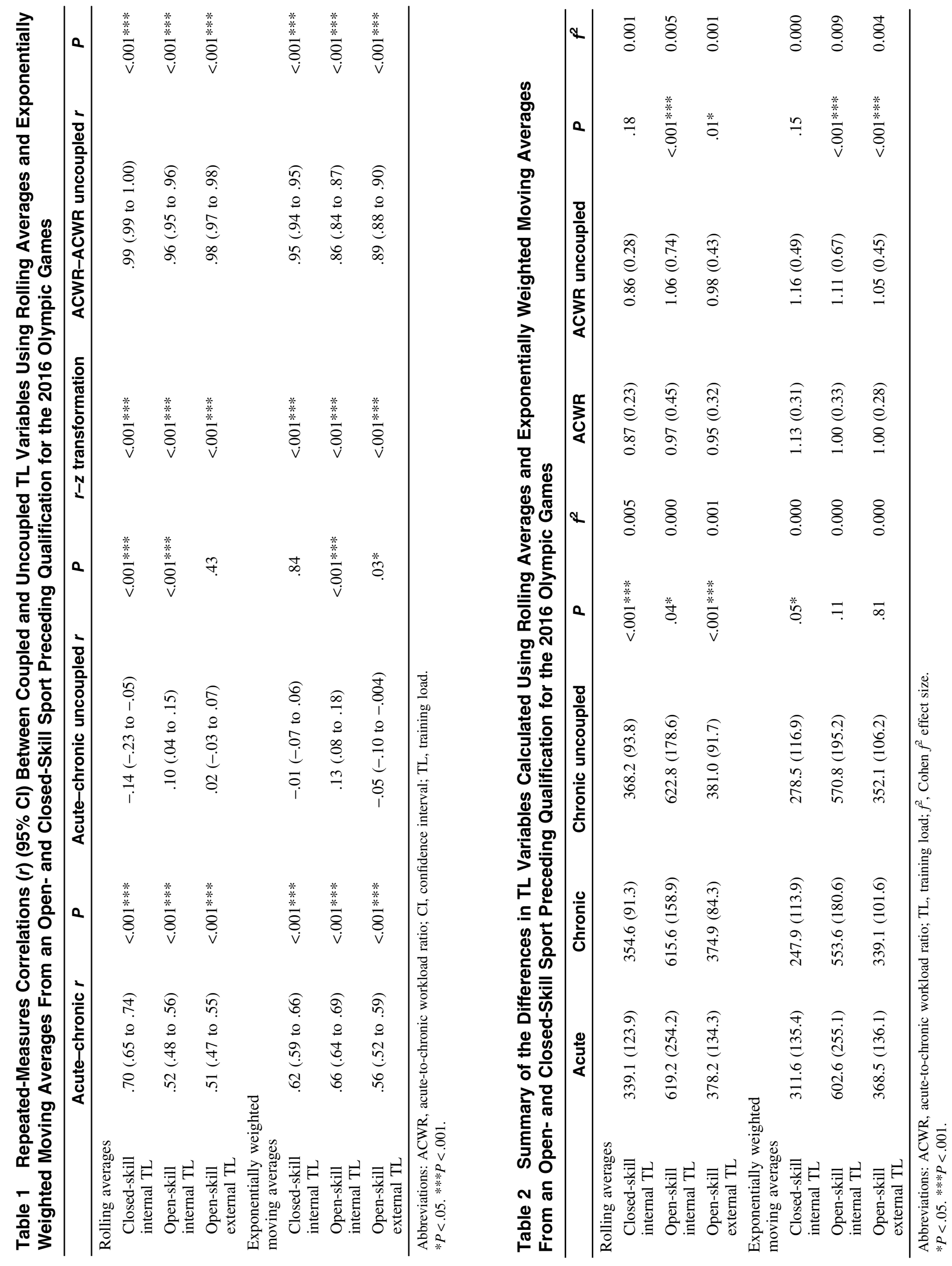

